\title{
Internet espace éditorial pour la communication homme-animal
}

\section{Lise Vieira}

\section{(2) OpenEdition}

1 Journals

Édition électronique

URL : http://journals.openedition.org/communicationorganisation/2832

DOI : 10.4000/communicationorganisation.2832

ISSN : $1775-3546$

Éditeur

Presses universitaires de Bordeaux

Édition imprimée

Date de publication : 1 mai 2003

ISSN : 1168-5549

Référence électronique

Lise Vieira, «Internet espace éditorial pour la communication homme-animal », Communication et organisation [En ligne], 23 | 2003, mis en ligne le 27 mars 2012, consulté le 04 mai 2019. URL : http:// journals.openedition.org/communicationorganisation/2832; DOI : 10.4000/ communicationorganisation.2832

Ce document a été généré automatiquement le 4 mai 2019.

(c) Presses universitaires de Bordeaux 


\title{
Internet espace éditorial pour la communication homme-animal
}

\author{
Lise Vieira
}

\section{Présentation du champ de recherche}

1 Les espaces numériques livrent à notre réflexion des objets d'autant plus délicats à observer qu'ils sont soumis à de constantes variations. Leur caractère composite contraint l'analyste s'il n'y était naturellement enclin, à la souplesse et à l'adaptabilité notamment en utilisant des méthodes et des outils empruntés à diverses spécialités scientifiques. Au carrefour de plusieurs disciplines, les Sciences de l'information et de la Communication sont rompues à ce genre d'exercice qu'elles pratiquent couramment dans leurs recherches épistémologiques. Les rapprochements entre Sciences exactes et Sciences humaines suscitent encore la critique voire l'ironie de maints spécialistes à ce point attachés à leurs cadres de références qu'on pourrait les soupçonner de confondre rigueur et rigorisme.

2 La nature même de la recherche scientifique particulièrement dans des domaines où prime l'innovation, nécessite la curiosité et l'esprit ouverture de façon à laisser à la démarche suffisamment d'interstices de liberté. C'est ce qu'Abraham Moles $^{1}$ évoque plaisamment sous le terme d'«adolescence des sciences » : constituées d'un champ de possibles, elles préservent une part d'incertitude. Dans son ouvrage La formation de l'esprit scientifique paru en 1951, Gaston Bachelard manifestait à ce sujet une certaine lucidité en affirmant : «L'homme animé par l'esprit scientifique désire sans doute savoir, mais c'est aussitôt pour mieux interroger $»^{2}$

3 C'est en effet la multiplication d'actions de recherche favorisant l'interaction entre disciplines - comme la journée d'études à laquelle nous participons aujourd'hui ${ }^{3}-$ qui permettra de mieux appréhender le réel mouvant qui nous entoure. 


\section{Liens de contiguiité entre la notion d'espace informationnel et la relation homme-animal}

\section{Premier lien de contiguïté}

4 Si l'on remonte aux sources du concept d'édition, on s'aperçoit que ce dernier trouve son origine dans la recherche et la transmission d'information, activité humaine aussi ancienne que l'existence même de notre espèce. Les besoins fondamentaux de nos ancêtres de la Préhistoire furent d'ordre vital : trouver abri et nourriture, se protéger contre des prédateurs de tous ordres, telle était leur quête quotidienne. Les dessins et gravures sur les parois des grottes, selon les recherches des plus éminents préhistoriens, avaient une fonction sacrée faisant partie d'un rituel destiné à invoquer des forces favorables à une chasse fructueuse, mais remplissaient aussi une fonction informative.

Les travaux d'André Leroi-Gourhand ${ }^{4}$ défendent la thèse selon laquelle Cro-Magnon projetait sur les parois de ces cathédrales souterraines que sont les grottes, ses croyances et ses connaissances. Les points, les bâtonnets et autres signes en forme de grille qui accompagnent (ou ponctuent) les fresques de taureaux et de chevaux sont considérés comme les prémices d'une écriture non encore déchiffrée à ce jour. Ainsi, à la racine même de l'activité humaine, on constate qu'il y a superposition de deux types de relation entre l'homme et l'animal. La représentation graphique de l'animal revêt une première fonction d'ordre pragmatique liée à l'activité de la chasse et à la nécessité de survie. Une seconde fonction manifeste un autre besoin non moins fondamental : celui de la mémoire. L'homme a très tôt éprouvé le besoin de laisser derrière lui des signes tangibles et intelligibles de son passage sur terre, ce souci de la trace étant une des caractéristiques fondatrices de l'entreprise éditoriale.

Edgard Morin exprime en ces termes cette thèse sur les prémices de l'écriture dans la préhistoire :

«Le champ graphique de l'humanité préhistorique est très vaste et très varié : y voisinent le signe conventionnel, le symbole plus ou moins analogique, la figuration extrêmement précise des formes vivantes et enfin la représentation d'êtres chimériques ou irréels. Il ne s'agit donc pas de nous interroger sur un art, la peinture, mais de tenter la graphologie de Sapiens... Le déploiement graphique constitue l'acquisition d'un nouveau mode d'expression et de communication qui est une première écriture $»^{5}$

\section{Second lien de contiguïté}

7 Il y a d'autre part une similarité entre le réseau des réseaux et certaines sociétés animales. La situation dans laquelle se trouve l'internaute ne manque pas en effet de rappeler les théories développées en biologie sur l'intelligence collective des insectes « sociaux » comme les fourmis ou les termites. Ainsi peut-on lire dans un article signé par Jean-Louis Deneubourg ${ }^{6}$ :

«Dans les sociétés d'insectes, le projet global n'est pas programmé explicitement chez les individus, mais émerge de l'enchaînement d'un grand nombre d'interactions élémentaires entre individus ou entre individus et environnement. Il y a en fait intelligence collective construite à partir de nombreuses simplicités individuelles ». 
Sans ordre apparent, composé d'innombrables unités interconnectées au milieu desquelles l'usager navigue sans pour autant avoir une représentation précise de leur totalité, on peut être tenté de comparer Internet à une vaste termitière. Cette analogie entre l'animal et l'humain, si elle a ses limites, a tout au moins pour vertu de situer nos actions et productions dans un ensemble plus vaste, dont la portée nous échappe en grande partie et qui appartient à la « logique du vivant $»^{7}$, ensemble éminemment ouvert et complexe. Elle replace l'humain dans son statut d'atome de l'univers et l'incite à une juste humilité. Dans son ouvrage Le Macroscope paru en 1975, Joël de Rosnay a apporté une large diffusion à la notion de système dont il souligne la complexité :

« Nous sommes confondus par le nombre et la prodigieuse variété des éléments, des relations, des interactions ou des combinaisons sur lesquels reposent le fonctionnement des grands systèmes dont nous sommes les cellules, pour ne pas dire les rouages. $\|^{8}$

En raison des nouvelles médiations de la connaissance qu'ils génèrent, ainsi que du maillage des contenus à l'aide des hyperliens, les réseaux numériques relèvent d'une logique d'ordre systémique. Ce courant de pensée, né il y a quelques décennies, concomitamment au sein de plusieurs disciplines ${ }^{9}$ a pour principe fondamental de considérer le tout plutôt que les parties ${ }^{10}$ » et donc de raisonner dans la globalité et la complexité des interelations ${ }^{11}$...

Les travaux de Pierre Lévy et particulier son ouvrage L'apparition de l'intelligence collective apportent un autre éclairage à ce propos : en portant en lui l'espoir d'une information universelle, le cyberespace apparaît comme la métaphore d'un hyper-cerveau humain, comme le lieu de la pensée collective de l'humanité. «L'intellectuel collectif est une sorte de société anonyme à laquelle chaque actionnaire apporte en capital ses connaissances, ses navigations, sa capacité d'apprendre et d'enseigner. Le collectif intelligent ne soumet ni ne limite les intelligences individuelles, mais au contraire les exalte, les fait fructifier et leur ouvre de nouvelles puissances. Ce sujet transpersonnel, ne se contente pas de sommer les intelligences individuelles. Il fait croître une forme d'intelligence qualitativement différente, qui vient s'ajouter aux intelligences personnelles, une sorte de cerveau collectif ou d'hypercortex $»^{12}$.

11 L'idée marquante que nous pouvons retenir de cette similitude entre les sociétés animales et humaines consiste à considérer la logique de type systémique comme un instrument particulièrement approprié pour appréhender les vastes ensembles informationnels qui nous entourent. La complexité de leurs innombrables unités en interaction, nous conduit à voir et à penser autrement et donc à trouver de nouvelles dynamiques de fonctionnement, afin d'accéder au changement.

\section{Le Web nouvel espace éditorial}

C'est cette notion de changement ou d'innovation définie par Patrice Flichy comme « rapprochement d'histoires parallèles, ajustement successif, confrontation et négociation, réduction de l'incertitude $»^{13}$ que nous allons maintenant explorer plus avant en l'appliquant au contexte de l'édition. Les réseaux numériques et particulièrement les sites Web d'Internet renouvellent le concept d'espace éditorial. Dans sa fonction première, l'acte d'édition ${ }^{14} \mathrm{se}$ fonde sur les notions de parution, publication et diffusion de contenus. Nous prendrons le terme d'espace éditorial dans son acception la plus large. 
13 Le web est un nouveau territoire permettant la mise en ligne de contenus qui jusqu'à un époque récente étaient essentiellement disponibles sur support papier (livres, revues, articles). Cette dimension est étroitement liée au concept traditionnel d'édition, qu'elle soit ou non à but lucratif : circuit commercial de l'édition ou publications à visée scientifique.

14 Mais dans une approche plus extensive, le web affirme aussi sa vocation éditoriale en offrant aux institutions et organisations quelles qu'elles soient un espace d'expression propre à renforcer leurs actions d'information et d'avoir une influence positive sur leur image et leur stratégie communicationnelle.

15 Nous appliquerons cette analyse à l'exemple des sites web traitant de la communication homme-animal.

\section{L'exemple des sites web traitant de la communication homme-animal}

\section{Méthodologie}

16 Les éléments que je viens de souligner mettent en évidence la nécessité de considérer le réseau numérique comme un espace d'expression éminemment novateur par rapport aux qualités comparées de l'édition numérique. Dans cette optique, j'ai choisi de ne pas recourir à une approche systématique des sites Web, mais d'en sélectionner quelques uns afin d'effectuer une analyse qualitative.

17 Une première exploration à l'aide des moteurs de recherche Google, Altavista et du répertoire Yahoo a consisté à appliquer deux requêtes en français et en anglais

18 - « communication-homme-animal - organisation »

19 (" communication-man-animal-organization »)

20 - « ethologie - homme - animal » (« ethologia- man- animal »)

21 Cela a permis de dégager parmi les résultats pertinents, une typologie des principaux sites abordant cette thématique :

22 - des sites de laboratoires scientifiques. Parmi les premières références figure très logiquement celle de l'appel à communication pour cette journée d'études sur la page du GREC/O : http://www.montaigne.u-bordeaux.fr/GRECO/actualité com.html

23 On peut également citer la présence du site scientifique LEEC (Laboratoire d'éthologie expérimentale et comparée de l'Université de Paris 13, auquel on accède facilement par la rubrique "biosociologie animale et humaine» de Yahoo (sur lequel nous reviendrons dans l'analyse) : http://leec.univ-paris13.fr

24 - des sites institutionnels d'associations œuvrant dans ce domaine. Par exemple le site de l'IAHAIO (association internationale s'occupant des interactions entre l'homme et l'animal) : http://users.skvnet.be/ethologia/Yahoo

25 - des sites de publications électroniques appartenant au circuit de la presse commerciale, comme celui du ${ }^{\circ} 108 \mathrm{du}$ journal Sciences Humaines :

http://www.scienceshumaines.fr/presomm.asp

27 ou encore celui du Nouvel Observateur : 

iconographiques) ligne, notamment : GÉNÉRALES SUR LE SITE

Nom du site

Adresse URL

Date de dernière mise à jour

Public cible

Accès au site : libre ou payant

\section{QUALITÉ ET FIABILITÉ DU CONTENU}

Abondance des ressources

Pertinence du contenu

Qualité et clarté des textes

\section{NAVIGATION ET ERGONOMIE}

Fonctionnalité des cadres

Qualité de l'image et du son

- Technologies utilisées (Flash...)

RÉFÉRENCEMENT ET ACCESSIBILITÉ

http://www.nouvelobs.com/articles/p 1931 /'a 1940.html

Pour une approche qualitative plus approfondie des sites web, j'ai retenu le principe de l'analyse des pages d'accueil, s'appuyant sur plusieurs grilles d'évaluation consultables en

http://www.rrsss06.gouv.qc.ca/english/commpub/publications/grille en.html

(Equipe régionale en documentation. Grille d'analyse de sites Web)

http://www.uco.fr/services/biblio/cdps/selec grille 4.html

(H. Basset. Grille d'évaluation des sites Web)

http://csidoc.insa-lvon.fr/sapristi/fristi36.html

(M. Joly, J.-M. Mermet. Evaluation de l'information présente sur Internet)

Les critères suivants sont communément retenus: IDENTIFICATION : INFORMATIONS

Options de langues pour l'interrogation du site

Types de d'information (documents intégraux, banques de données (textuelles ou

Médias mis en œuvre : multimédia texte-image son ou texte illustré ou texte seul

Présence et pertinence des illustrations

Droits d'auteur et sources (identification des ressources)

Qualité et pertinence des liens internes et externes

Facilités de navigation (retour à la page d'accueil, plan du site)

Fonctionnalité claire des boutons de navigation

\section{PRÉSENTATION GRAPHIQUE ET SONORE}

Lisibilité : choix des couleurs et des caractères, densité visuelle du texte

Rapidité de chargement des images et sons

Site référencé dans les principaux moteurs de recherche 


\section{Enjeux et perspectives} représentent la diffusion de contenus sur Internet concernent tous les types d'organisation qu'elles soient à vocation économique ou à portée plus symbolique. Nous les aborderons selon trois points de vue :

\section{Les enjeux scientifiques et éditoriaux}

En passant de l'imprimé au numérique ${ }^{16}$, on voit perdurer les fonctions éditoriales fondamentales de parution, publication et diffusion de contenus, tout en en voyant poindre de nouvelles caractéristiques.

Du point de vue de l'émetteur, la publication sur Internet pose des difficultés d'ordre juridique, technique et économique. Certains décideurs craignent encore pour la protection de l'œuvre et des droits d'auteur dans un secteur animé d'autre part par une forte concurrence internationale ${ }^{17}$. En outre, l'élaboration d'un produit multimédia demande des compétences spécifiques en informatique et en architecture logicielle. Malgré cela, les publications en ligne se multiplient, en particulier sous forme de revues électroniques. Ce mode de diffusion permet en effet de faire baisser les coûts de production d'environ $30 \%$, puisqu'on évite les frais de tirage en de multiples exemplaires et que l'on réduit ceux occasionnés par le stockage et par la distribution.

Parmi les innombrables pages qui transitent sur les réseaux, beaucoup trop d'entre elles ne peuvent se dégager des modèles antérieurs et s'inspirent encore largement de l'imprimé. Mettre en forme des données numériques ne revient pas à les reproduire telles qu'elles se présentent sur les supports papier ${ }^{18}$. D'où la nécessité de prendre en compte outre la notion d'architecture fonctionnelle du site, ainsi que des paramètres afférant au graphisme et à l'ergonomie ${ }^{19}$. De la capacité à accepter la nouvelle donne dépend en grande partie l'avenir de l'édition électronique commerciale.

76 Pour le chercheur, le réseau Internet présente l'indéniable avantage de diminuer les délais de publication souvent fort longs dans les revues papier. On voit ainsi de développer un circuit parallèle de diffusion de contenus scientifiques dont les produits court-circuitent désormais les mécanismes commerciaux traditionnels. La garant de la qualité scientifique n'est alors plus le comité éditorial de la revue mais le laboratoire de recherche hébergeant cette production. 


\section{Les enjeux cognitifs} fonctions à portée stratégique différenciée. Le premier niveau stratégique concerne la fonction de visibilité : c'est l'effet vitrine » qui incite l'organisation à être présente sur le Web pour occuper un territoire au même titre que la concurrence.

81 A un second niveau, cette présence peut revêtir une autre visée stratégique : le caractère novateur de la technologie numérique a un effet positif sur l'image de l'entreprise par simple effet de proximité. L'utilisation les TIC ne peut en effet que connoter le dynamisme et l'adhésion au changement.

En troisième point, on peut postuler que l'appropriation par une organisation de technologies numériques comme l'Internet et l'Intranet a une influence sur le mode même de fonctionnement de cette organisation. On passe en effet d'un modèle de circulation d'information de type descendant, arborescent et hiérarchique à un autre modèle qui est celui d'une information multidirectionnelle et réticulée. L'information en réseau, par définition mettant en place de multiples interconnexions entre les données, cela peut entraîner de profonds changements structurels dans cette entreprise ou organisme. 

capacité qu'elles offrent en matière d'interactivité. La communication électronique bouleverse le schéma traditionnel du récepteur cantonné à sa place de récepteur passif : on passe du "un vers tous» (" one to many») des médias traditionnels au «tous vers tous » (" many to many») du cyberespace. Il s'agit là d'un processus de rétroaction qui ne peut qu'optimiser le processus de communication: non seulement l'implication du visiteur dans l'élaboration d'un site est susceptible d'assurer sa fidélisation, mais elle peut en outre contribuer à l'amélioration du contenu en le rendant plus proche des attentes du public cible.

\section{Perspectives} des organisations et celles qui s'intéressent à la communication homme-animal se trouvent particulièrement dans cette mouvance, puisqu'elles se situent dans une perspective éminemment novatrice permettant la confrontation de points de vue diversifiés. On peut remarquer que cette démarche intellectuelle est métaphorique du réseau, lui même système interrelationnel.

Compris comme espace éditorial innovant, Internet génère la création de produits à valeur ajoutée dont les principales caractéristiques sont la disponibilité, la transparence et l'interactivité. Quelle que soit sa nature ou sa vocation, toute organisation décidée à intégrer la mouvance des évolutions technologiques se doit de prendre en compte les capacités techniques et conceptuelles du numérique. Internet et les réseaux électroniques posent de manière différente, sans pour autant les abolir, les notions de temps et de distance. Dans le contexte de surproduction économique et informationnelle qui est le propre de notre monde actuel, connectivité, vitesse et immatérialité deviennent des enjeux fondamentaux.

\section{BIBLIOGRAPHIE}

BACHELARD, G., La formation de l'esprit scientifique. Paris : Gallimard, 1951.

DENEUBOURG, J.-L., Individuellement, les insectes sont bêtes. Collectivement, ils sont intelligents. Le Temps stratégique n 65. Genève, septembre 1995.

Texte en ligne : http://vvww.archipress.org/ts/deneubourg.htm.

FLICHY, P.. L'innovation technique. Récents développements en sciences sociales : vers une nouvelle théorie de l'innovation. Paris : Éditions de la Découverte, 1995.

JACOB, F., La Logique du vivant. Paris : Gallimard, 1976, 352 p. (Coll. Tel) LEROI-GOURHAN, A., La Préhistoire de l'art occidental. Paris : Mazenod. 1968. (coll. Arts et grandes civilisations)

LEVY, P., L'apparition de l'intelligence collective. Pour une anthropologie du cyberespace. Paris : La Découverte, 1994.

Communication et organisation, 23 | 2003 
MOLES, A., Les Sciences de l'imprécis. Chapitre La science en train de se faire. Paris : Éditions du Seuil, 1995. p. 33 (coll. Points)

MORIN. E., Le paradigme perdu : la nature humaine. Paris : Éditions du Seuil, 1973. (coll. Points)

QUEAU, P., Le virtuel. Vertus et vertiges. Paris : Champ Vallon. 1993 ROSNAY, J. (de), L'Homme symbiotique. Paris : Le Seuil, 2000 (nouvelle édition), $432 \mathrm{p}$.

ROSNAY, J. (de), Le Macroscope. Paris : Le Seuil, 1975. (coll. Points)

SCHUWER, P., Article « Édition » de l'Encyclopaedia Universalis

SCHUWER. P., Traité pratique d'édition. Paris : Electre-Editions du Cercle de la librairie, 1997.

VIEIRA, L., De l'imprimé au numérique. L'édition électronique, enjeux et stratégies pour la diffusion de l'information. Synthèse présentée pour l'Habilitation à diriger les recherches. Université de Bordeaux 3-Michel de Montaigne. Directeur Roland Ducasse. Janvier 1999, 325 p.

VIEIRA, L., Évolution des formes d'édition dans le contexte multimédia. Perspectives pour l'enseignement Supérieur et la recherche. Colloque GRESIC. La Communication de l'information Scientifique et Technique dans l'Enseignement Supérieur et la recherche Bordeaux 16-18 mars 1995. Paris : ADBS. 1995. p. 81-87.

WATZLAWICK, P.. HELMICK, B. J., JACKSON, D. D., Une logique de la communication. Paris : Éditions du Seuil, 1979. (Coll. Points)

\section{NOTES}

1. Abraham Moles. Les Sciences de l'imprécis. Chapitre «La science en train de se faire. Paris: Éditions du Seuil. 1995. P. 33 (coll. Points)

2. Gaston Bachelard. La formation de l'esprit scientifique. Paris : Gallimard. 1951. p. 16

3. Notons que cette thématique suscite également l'intérêt d'un plus large public, puisque dans la semaine où s'est déroulée la journée d'études organisée par le CEDHET, deux émissions ont été programmées : Mardi 4juin, sur France3 Vie Privée vie publique: Jusqu'où est on prêt à aller pour nos amis les bêtes ?.http://www.fr3.fr et Jeudi 6 juin sur France 5: Le Génie animal Révolutionner l'intelligence artificielle. http//.www.fr.fr

4. Cf. les travaux d'André Leroi-Gourhan. La Préhistoire de l'art occidental. Paris : Mazenod. 1968. (coll. Arts et grandes civilisations)

5. Edgar Morin. Le paradigme perdu : la nature humaine. Paris : Editions du Seuil. 1973. (coll. Points)

6. Jean-Louis Deneubourg. Individuellement, les insectes sont bêtes. Collectivement, ils sont intelligents. Le Temps stratégique, $\mathrm{n}^{\circ} 65$. Genève. Septembre 1995. Texte en ligne: http:// www.archipress.org/ts/deneuhourg.htm.

7. Francois Jacob. La Logique du vivant. Paris : Gallimard. 1976. 352 p. (Coll. Tel)

8. Joël de Rosnay. Le Macroscope. Paris : Le Seuil. 1975. (coll. Points) Voir aussi Josi de Rosnay. L'Homme symbiotique. Paris : Le Seuil. 2000 (nouvelle édition). $432 \mathrm{p}$.

9. En particulier la cybernétique, initiée par Norbert Wiener

10. La théorie allemande de la Gestalt a. en son temps, apporté une contribution majeure en ce sens, puisqu'elle avance que le tout est différent de la somme de ses parties, que ce dernier est indépendant par rapport à la nature de ses parties et qu'il est donc porteur d'une connaissance qui lui est propre

11. Gregory Bateson et Paul Watzlawick ont appliqué dans leurs travaux à l'école de Palo Alto cette notion d'interrelation au domaine des Sciences Humaines. Ils ont montré que la communication, loin de se borner à une simple transmission d'informations, influe sur la 
manière de recevoir le message et le comportement des usagers : elle est porteuse de liens sociaux.

Paul Watzlawick. Janet Helmick Beavin. Don D. Jackson. Une logique de la communication. Paris : Editions du Seuil. 1979. (Coll. Points). Titre original : Pragmatics of Human Communication. A Study of Interactional Patterns. Pathologies and Paradoxes

12. Pierre Lévy. L'apparition de l'intelligence collective. Pour une anthropologie du cyberespace. Paris : La Découverte. 1994.

13. Patrice Flichy. L'innovation technique. Récents développements en sciences sociales: vers une nouvelle théorie de l'innovation. Paris : Éditions de la Découverte. 1995. p. 236

14. Philippe Schuwer. Traité pratique d'édition. Paris : Electre-Editions du Cercle de la librairie. 1997. p 7.

Lire par ailleurs Philippe Schuwer, article « Edition » de l'Encyclopaedia Universalis

15. La présentation orale de ce travail a permis d'illustrer le propos en présentant plusieurs exemples de pages d'accueil de sites ayant pour point commun la thématique de la communication homme-animal. En particulier :

http ://www.montaigne.u-bordeaux.rr/GRECO/actualite corn.html

http ://leec.univ-paris13.fr

http ://users.skvnet.be/ethologic/yahoo

http:/www.scienceshumaines.rr/presomm.asp

http ://www.nouvelobs.eom/articles/p 1931 /a 1940.html

http ://WWW.afirac.com

16. Lise Vieira. De l'imprimé au numérique. L'édition électronique, enjeux et stratégies pour la diffusion de l'information. Synthèse présentée pour l'habilitation à diriger les recherches. Université de Bordeaux 3-Michel de Montaigne. Directeur Roland Ducasse. Janvier 1999. 325p.

17. Elle est orchestrée par les grands groupes internationaux (Reed Elsevier. Springer Verlag. Vivendi Universal...)

18. Lise Vieira. Evolution des formes d'édition dans le contexte multimédia. Perspectives pour l'enseignement Supérieur et la recherche. Colloque GRES1C. La Communication de l'information Scientifique et Technique dans l'Enseignement Supérieur et la recherche Bordeaux 16-18 mars 1995. Paris : ADBS. 1995. p. 81-87

19. Ce concept se définit comme «La mise en œuvre de connaissances scientifiques relatives à l'homme et nécessaires pour concevoir des outils, des machines et des dispositifs qui puissent être utilisés par le plus grand nombre avec le plus de confort, de sécurité et d'efficacité ».R Rubio. Vergonome.com : l'ergonomie du Web. Décembre 2000.

http ://www. lergonome.com/dev/pages/article__Lasp\#TOP

20. Philippe Quéau. Le virtuel. Vertus et vertiges. Paris : Champ Vallon. 1993

\section{RÉSUMÉS}

Au-delà d 'une typologie des principaux sites Web traitant de la relation Homme/animal, nous tenterons de dégager des tendances parmi leurs pratiques éditoriales afin de déceler le caractère novateur de leurs productions. Il s'agit de mesurer l'enjeu que peut représenter la production électronique d'un point de vue stratégique. Rares sont les institutions qui ne possèdent désormais leur site Web, mais la question fondamentale est d'estimer l'usage qu'elles en font. Les 
potentiels du numérique modifient les modalités de conception, de diffusion et de réception des messages: Internet est un média de diffusion, mais aussi d'interaction. Quels enjeux cela représente-t-il pour la communication des organisations qui s'intéressent au phénomènes d'interaction entre l'homme et l'animal ?

After drawing a typology of the main Web sites dealing with the relation between man and animals (corporate information, factual data, articles and texts...) the author tries to distinguish what are the trends in publishing activities, and see how much innovative they are. The aim is to measure what strategic dimension is at slake in electronic publishing. Most of institutions now display a Web site, but the question remains of what they really do with it. Technical potentialities of numerical encoding induce new modes of conception, diffusion and reception of messages. Internet is both a diffusion and an interaction medium. What use do organizations for social, economical, political or health action in the field of man/animal interaction make of this potential?

\section{INDEX}

Mots-clés : internet, diffusion de l'information, numérisation, média, fonction éditoriale

\section{AUTEUR}

\section{LISE VIEIRA}

Lise Vieira enseigne les sciences de l'information et de la communication à l'Université Bordeaux 3. Elle est titulaire d'une habilitation à diriger les recherches, avec pour sujet : De l'imprimé au numérique. L'édition électronique, enjeux et stratégies pour la diffusion de l'information. 